\title{
CORRESPONDENCE Induction of human dendritic cell maturation by naïve and memory B-cell subsets requires different activation stimuli
}

\author{
Cellular \& Molecular Immunology (2018) 15:1074-1076; https://doi. \\ org/10.1038/s41423-018-0017-z
}

Dendritic cells (DCs) are the master regulators of the immune response and engage in constant cross-talk with other immune cells to initiate an appropriate immune response. The outcome of cross-talk with various immune cells towards DC functions has been shown to be dependent on the particular subset of cells and their activation stimuli. Here we show that for human $B$ cells to induce maturation of DCs with an enhanced T-cell stimulatory capacity, B-cell receptor (BCR) signaling is sufficient for memory B-cells, whereas additional CD40 signaling is required for naïve $B$ cells to induce complete activation of DCs.

DCs are the most efficient antigen-presenting cells and play a crucial role in the initiation and regulation of the immune response. This unique function of DCs is attributed to their capacity to recognize insults from their surroundings and via cross-talk with other cells and soluble immune mediators, including cytokines and antibodies. ${ }^{1-3}$ In fact, DCs engage in a dialog with various immune cells, including $T$ cells, NK cells, and B cells, which can influence the quality of the immune responses induced by DCs. Furthermore, different subset of cells within a given immune cell population can have a distinct impact on the functional status of DCs. Accordingly, naïve and effector memory $T$ cells induce the maturation of DCs with a potent T-cell stimulatory capacity, whereas regulatory $T$ cells (Treg cells) impart a tolerogenic feature on DCs. ${ }^{4}$

In addition to T cells, B cells also have a distinct ability to regulate the function of DCs. Thus, B cells activated via toll-like receptor 9 (TLR9) signaling either alone or in combination with CD40 stimulation, which are the stimuli that have been shown to promote regulatory B cells (Breg cells), ${ }_{1}^{5}$ were found to restrict the maturation of human DCs. ${ }^{6}$ On the contrary, our previous results indicated that the regulation of human DCs by $B$ cells depends on the signals that they receive. ${ }^{7}$ In agreement with this finding, we showed that through contact-dependent interaction, B cells that were activated via BCR (with or without CD40 signaling) induce maturation of DCs. ${ }^{8}$ These findings suggest that the B-cell-mediated regulation of DC function may depend on the activation signals received by $B$ cells.

Like $\mathrm{CD}^{+}{ }^{+} \mathrm{T}$ cells, B cells are highly heterogeneous. Naïve and memory B cells represent major B-cell subsets and are distinct in their phenotypic and functional features. ${ }^{9,10}$ Because of previous antigen experience, memory $B$ cells, in general, are known to respond rapidly and robustly to activation stimuli. ${ }^{11}$ B-cell subsets also have diverse impacts on anti-tumor immunity. ${ }^{12}$ Considering these distinct properties of naïve and memory B-cell subsets, we investigated whether B-cell subsets have different abilities to regulate human $D C$ functions.

\section{BCR-ACTIVATED MEMORY, BUT NOT NAÏVE B CELLS, INDUCE DC MATURATION}

We first investigated the effect of BCR-activated naïve and memory B cells on the phenotype of DCs that were differentiated from peripheral blood monocytes of healthy donors' buffy bags, as described previoulsy. ${ }^{8}$ Permission from the ethics committee was obtained for the use of these materials for research purposes (Institut National de la Santé et de la Recherche-EFS ethical committee convention 15/EFS/012). Pure CD19 ${ }^{+}$B cells were isolated from peripheral blood mononuclear cells by negative selection process by using the $B$ cell isolation kit II, human (Miltenyi Biotec, Paris, France). CD27 microbeads were used to separate naïve $\left(C D 27^{-}\right)$and memory $\left(C D 27^{+}\right)$B-cell subsets from these $B$ cells. The naïve and memory B-cell subsets $\left(0.5 \times 10^{6}\right.$ cells/ $\mathrm{mL}$ ) were pre-activated with $B C R$ alone using a $F\left(a b^{\prime}\right)_{2}$ of goat antihuman IgM $(10 \mu \mathrm{g} / \mathrm{mL}$; Jackson ImmunoResearch Laboratories, West Grove, USA) or with BCR + CD40 stimulation using goat antihuman $\operatorname{lgM}$ and an agonistic anti-human CD40 monoclonal antibody (mouse lgG2B clone 82111, R\&D Systems, Lille, France; $5 \mu \mathrm{g} / \mathrm{mL}$ ) for $48 \mathrm{~h}$.

Immature DCs $\left(0.1 \times 10^{6}\right.$ cells $/ 200 \mu \mathrm{L} /$ well $)$ were seeded in U-bottom 96-well plates with GM-CSF and IL-4 and were cultured either alone as control $\mathrm{DCs}\left(\mathrm{DC}_{\mathrm{ctrl}}\right)$ or co-cultured at a 1:1 ratio with $\mathrm{CD} 27^{-}$naïve $\mathrm{B}$ cells or $\mathrm{CD} 27^{+}$memory $\mathrm{B}$ cells that were preactivated by $B C R$ ( $D C_{\text {Naiive-B-pBCR }}$ or $D C_{\text {Memory-B-pBCR }}$ ) stimulation (Fig. 1a). Prior to co-culture with immature DCs, pre-activated $B$ cells were treated with mitomycin $C\left(50 \mu \mathrm{g} / 0.5 \times 10^{6}\right.$ cells $/ \mathrm{mL}$; Sigma-Aldrich, Saint-Quentin Fallavier, France) and were washed thoroughly to generate mitotically inactive cells and to keep the B cell number constant in the B cell-DC co-culture systems.

After $48 \mathrm{~h}$ of culture, the cells were harvested, and supernatants were collected for the analysis of IL-6 and CCL22 (DuoSet ${ }^{\circledR}$ ELISA kit, R\&D Systems). The phenotype of the DCs was analyzed by flow cytometry after staining with fluorochrome-conjugated antibodies (FITC-conjugated antibodies to CD1a, CD86, HLA-DR; PEconjugated antibodies to CD83, CD80, from BD Biosciences, Paris, France and anti-CD40-PE from Beckman Coulter, Villepinte, France).

We found that BCR-activated naive $B$ cells did not significantly modify the phenotype of immature $D C s\left(D C_{\text {Naive- } B-p B C R}\right)$ and that the expression of various DC markers was similar to that of control DCs ( $\left.C_{c t r l}\right)$ (Fig. 1b-d). These results indicate that BCR-activated naïve $B$ cells do not provide sufficient signals for $D C$ maturation. Interestingly, memory $B$ cells activated by BCR signaling induced

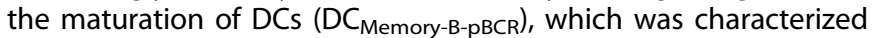
by significantly enhanced expression of co-stimulatory molecules, including CD80, CD86 and CD40; the antigen-presenting molecule HLA-DR, and the terminal maturation marker CD83 (Fig. 1b-d). Furthermore, only BCR-activated memory cells but not naïve $B$ cells were able to induce significant levels of cytokines (IL-6) and chemokines (CCL22) in DCs (Fig. 1e). Together, these data demonstrate that BCR-mediated activation signals allow memory $B$ cells but not naïve $B$ cells to induce the maturation of $D C s$. 

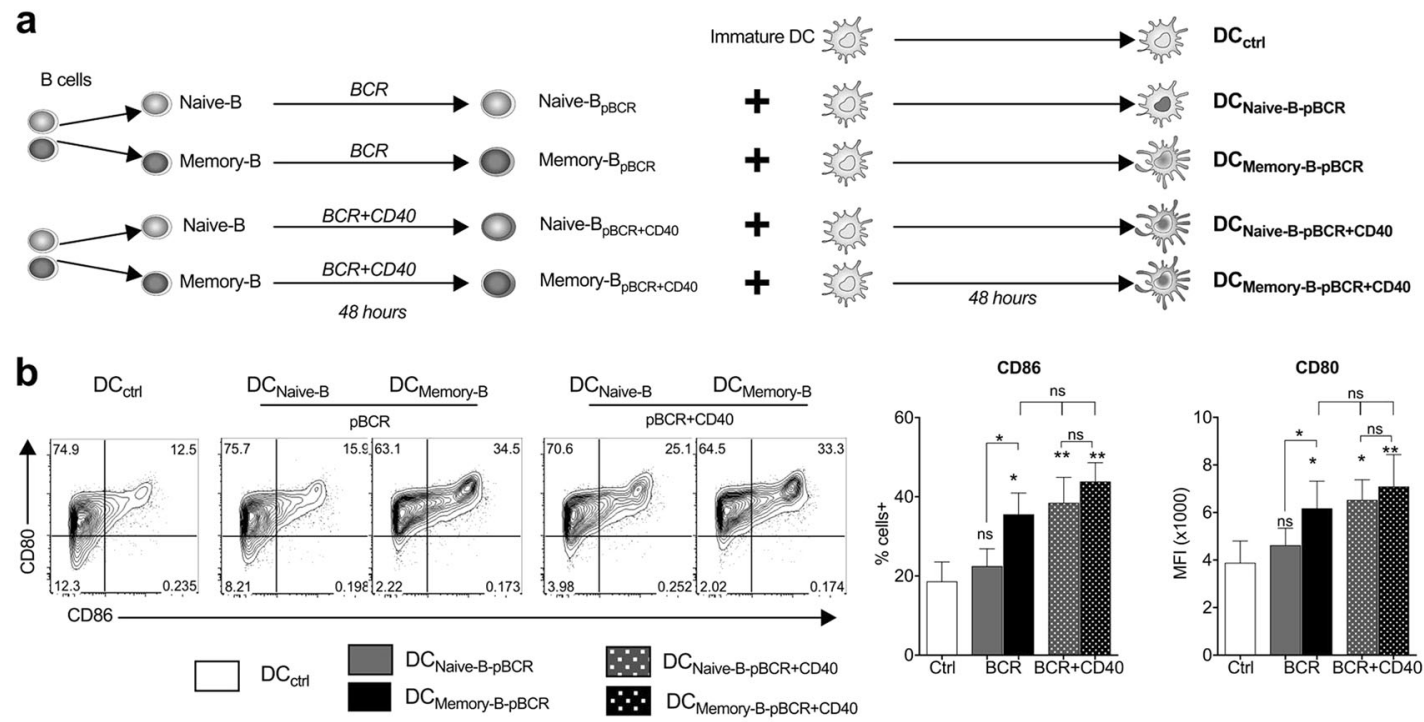

C
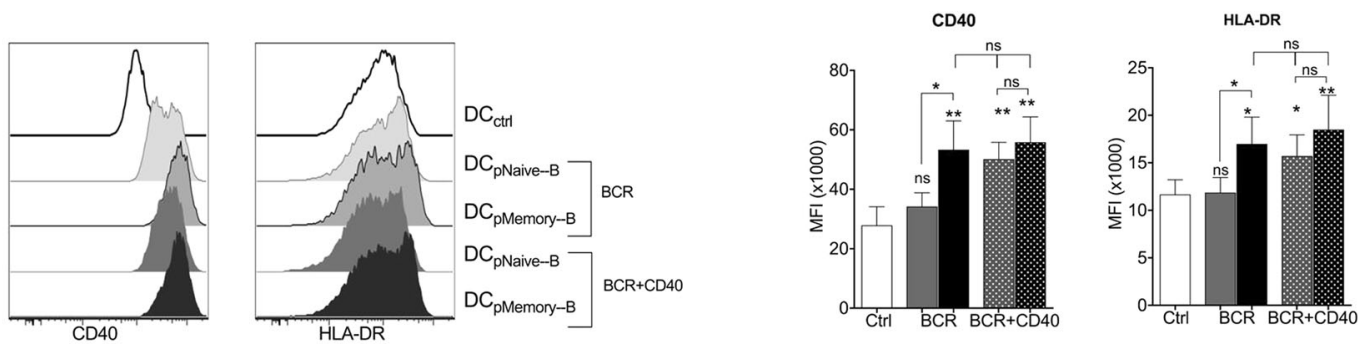

d
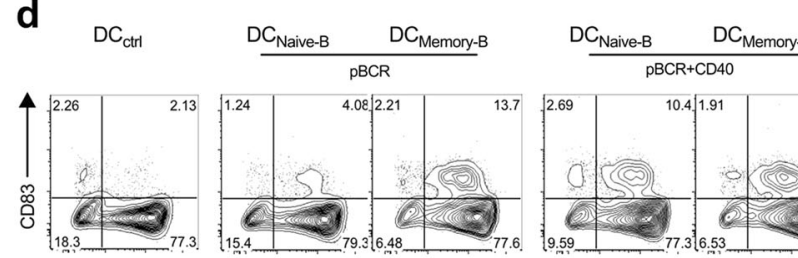
CD1a

$\square \mathrm{DC}_{\mathrm{ctrl}}$

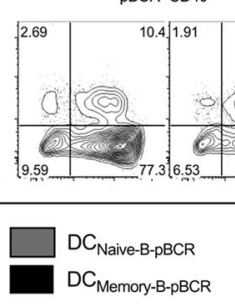

\footnotetext{
(1)
}
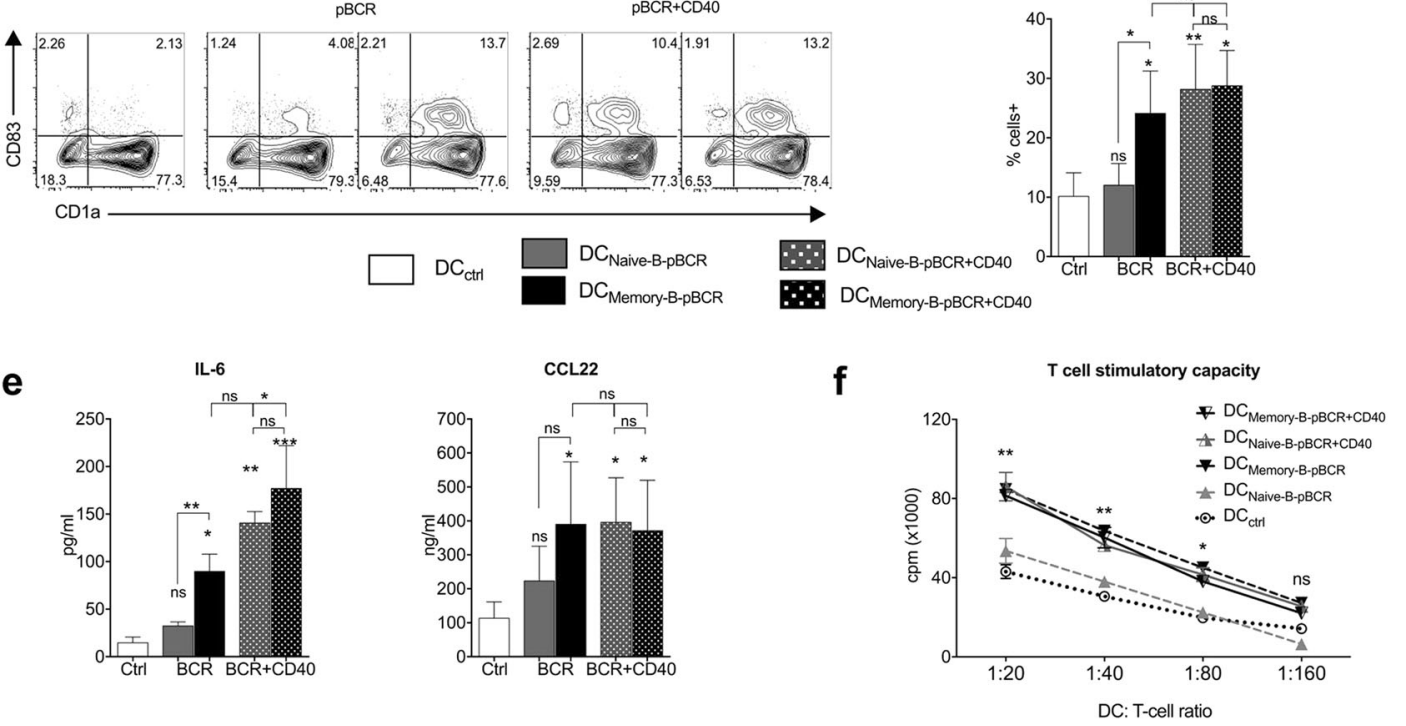

Fig. 1 Different requirements of the activation stimuli for naive and memory $B$ cells to induce the maturation of human dendritic cells. a Experimental design. Immature DCs were cultured in medium containing GM-CSF and IL-4 for $48 \mathrm{~h}$ either alone (DC $\left.\mathrm{Ctr}_{\mathrm{c}}\right)$ or co-cultured at a $1: 1$ ratio with $C D 27^{-}$naïve $B$ cells or $C D 27^{+}$memory $B$ cells that were pre-activated by $B C R\left(D C_{\text {Naiive-B-pBCR or }} D C_{\text {Memory-B-pBCR }}\right.$ ) or $B C R+C D 40$ $\left(D C_{\text {Naiive-B-pBCR }+C D 40 \text { or }} C_{\text {Memory-B-pBCR }+C D 40}\right.$ ) stimulation. b-d Phenotypic analysis (\% positive cells and mean fluorescence intensity MFI) of DCs for the expression of co-stimulatory (CD80, CD86, CD40) and antigen-presenting (HLA-DR) molecules and the maturation marker (CD83). Representative plots and the mean \pm SEM of data from four donors. e The supernatants of DC-B cell co-cultures were analyzed for the levels $(\mathrm{pg} / \mathrm{mL})$ of the IL- 6 cytokine and CCL22 chemokine (mean \pm SEM, $n=4$ donors). $\mathrm{f}$ DCs were purified from B cell-DC co-cultures by depleting B cells using CD20 microbeads. CD4 ${ }^{+}$T cells were co-cultured with DCs at various ratios for 5 days. DC-mediated CD4 ${ }^{+} \mathrm{T}$-cell proliferation was determined by the $\left[{ }^{3} \mathrm{H}\right]$ incorporation assay. Values are presented as counts per minute $(\mathrm{cpm})$. Data are the mean \pm SEM from quadruplicate wells and are representative of two independent experiments. ${ }^{*} P<0.05 ;{ }^{*} P<0.01 ;{ }^{* *} P<0.001$; ns not significant by one way-ANOVA 


\section{CD40 SIGNALING PERMITS BCR-ACTIVATED NAÏVE B CELLS TO INDUCE THE MATURATION OF DCS}

Previous studies have shown that naïve and memory B cells differ in their response to $B C R$ signaling $^{9}$ and in their requirements for their differentiation into antibody-secreting plasma cells. ${ }^{11}$ Therefore, we explored whether additional activation stimuli permit naïve $B$ cells to induce DC maturation. To address this question, naïve and memory $B$ cells pre-activated with $B C R$ and CD40 stimuli (BCR + CD40) were co-cultured with immature $D C s\left(D C_{\text {Naïve-B-pBCR }+C D 40}\right.$ or $\left.D C_{\text {Memory-B-pBCR }+C D 40}\right)$. We found that $D C s$ co-cultured with $B C R+C D 40$-activated naïve $B$ cells $\left(D C_{\text {Naiive-B-pBCR }+C D 40}\right)$ exhibited phenotypic changes suggestive of maturation compared to control DCs (DC $\left.C_{c t r l}\right)$ (Fig. 1b-d). In agreement with this finding, increased levels of cytokines and chemokines were also secreted by these DCs (Fig. 1e). Interestingly, expression of activation markers and production of IL-6 and CCL22 by DCs cultured with BCR + CD40-activated memory B cells $\left(D C_{\text {Memory-B-pBCR+CD40 }}\right.$ ) were similar to those of $B C R+C D 40-$ activated naive $B$ cells (Fig. $1 \mathrm{~b}-\mathrm{d}$ ). These findings suggest that while BCR signaling is adequate for memory $B$ cells to induce DC maturation, additional CD40 signaling is required for naïve $B$ cells to induce the maturation of DCs and to stimulate the production of DC cytokines.

\section{BCR SIGNALING IS SUFFICIENT FOR MEMORY B CELLS TO INDUCE THE MATURATION OF DCS WITH T-CELL STIMULATORY CAPACITY}

One of the main functions of DCs is to stimulate a T cell response. Therefore, we examined whether differences in the ability of B cell subsets to induce DC maturation are also reflected in their ability to regulate the T-cell stimulatory ability of DCs. Following $48 \mathrm{~h}$ of B cell-DC co-culture, B cells were depleted using CD20 microbeads, and B cell-depleted DCs were used for co-culture with $\mathrm{CD}^{+}$ T cells. The purity of DCs following B cell depletion was up to $98 \%$. $\mathrm{CD}^{+} \mathrm{T}$ cells were positively selected using $\mathrm{CD} 4$ microbeads (Miltenyi Biotec). For a mixed lymphocyte reaction, graded doses of B cell-depleted DCs were co-cultured in a U-bottom 96-well plate with allogeneic $C D 4^{+}$T cells $\left(0.05 \times 10^{6}\right.$ per well $\left./ 200 \mu \mathrm{L}\right)$ in complete RPMI-1640 medium supplemented with $10 \%$ human $A B$ serum. After 4 days in culture, cells were pulsed with $1 \mu \mathrm{Ci}(0.037$ $\mathrm{MBq})\left[{ }^{3} \mathrm{H}\right]$ thymidine overnight to quantify T-cell proliferation. Radioactive incorporation was measured on the fifth day using standard liquid scintillation counting, and the results were expressed as counts per minute.

In agreement with the phenotypic profiles, naïve $B$ cells activated with $B C R$ signaling did not alter the capacity of DCs ( $\left(D C_{\text {Naiive-B-pBCR }}\right)$ to induce $T$ cell proliferation, which was similar to that of control DCs $\left(D C_{\mathrm{ctrl}}\right)$.

On the contrary, memory $B$ cells that received either $B C R$ signaling alone ( $\left(\mathrm{C}_{\text {Memory-B-pBCR}}\right)$ or $\mathrm{BCR}+\mathrm{CD} 40$ stimulation $\left(D C_{\text {Memory-B-pBCR+CD40 }}\right.$ ) significantly enhanced the ability of DCs to induce the proliferation of $T$ cells (Fig. 1f). Taken together, these results indicate that $B C R$ signaling is sufficient for memory $B$ cells to induce the maturation of DCs with enhanced T-cell stimulatory capacity, whereas $B C R+C D 40$ signaling is required for naïve $B$ cells.

B cells play a diverse role during an immune response. In addition to producing antibodies, which are immune response modifiers, B cells also exert antibody-independent regulatory and effector functions, including antigen presentation to $T$ cells and the production of cytokines and chemokines. Interestingly, our recent findings show that, similar to $T$ cells, NK cells, and neutrophils, B cells also positively regulate the functional status of DCs in a contact-dependent manner. Furthermore, the present study reveals that naive $B$ cells require antigen recognitioninduced BCR signaling and T-cell-induced CD40 signaling to induce DC maturation, whereas memory B cells acquire this competency following BCR signaling, even in the absence of CD40 signaling. This finding may be due to the rapid response of memory B cells to activation stimuli and to upregulate surface markers involved in the interaction with DCs. ${ }^{9,10,13}$ Our findings are relevant for chronic inflammatory conditions, such as allergy and autoimmune diseases. Here, memory B cells can be activated, even in the absence of $T$ cells, by sensing persistent (self) antigens. These activated B cells, in turn, gain the ability to activate DCs to induce a pathogenic T-cell response and antibody production (autoantibodies and allergenspecific $\lg \mathrm{E}$ ) to further enhance an ongoing destructive immune response.

\section{ACKNOWLEDGEMENTS}

Supported by the Institut National de la Santé et de la Recherche Médicale (INSERM), Sorbonne Université and Université Paris Descartes, Paris, France.

\section{AUTHOR CONTRIBUTIONS}

M.S.M. and J.B. designed the study. M.S.M. performed the experiments. M.S.M., S.V.K., and J.B. analyzed the data. M.S.M. and J.B. drafted the manuscript.

\section{ADDITIONAL INFORMATION}

Competing interests: The authors declare no competing interests.

$$
\begin{array}{r}
\text { Mohan S. Maddur } \mathbb{D}^{1,2,3,5} \text {, Srini V. Kaveri }{ }^{1,2,3,4} \text { and } \\
\text { Jagadeesh Bayry }
\end{array}
$$

${ }^{1}$ Institut National de la Santé et de la Recherche Médicale, Paris F75006, France; ${ }^{2}$ Centre de Recherche des Cordeliers, EquipeImmunopathologie et Immunointervention Thérapeutique, Paris F75006, France; ${ }^{3}$ Sorbonne Université, Paris F-75006, France and ${ }^{4}$ Université Paris Descartes, Sorbonne Paris Cité, Paris F-75006, France ${ }^{5}$ Present address: Pfizer, Inc., Pearl River, New York, NY, USA

\section{REFERENCES}

1. Banchereau, J. et al. Immunobiology of dendritic cells. Annu Rev. Immunol. 18, 767-811 (2000).

2. Maddur, M. S., Kaveri, S. V. \& Bayry, J. Circulating normal IgG as stimulator of regulatory T cells: lessons from intravenous immunoglobulin. Trends Immunol. 38, 789-92 (2017).

3. Liu, J. \& Cao, X. Cellular and molecular regulation of innate inflammatory responses. Cell. Mol. Immunol. 13, 711-21 (2016).

4. Veldhoen, M., Moncrieffe, H., Hocking, R. J., Atkins, C. J. \& Stockinger, B. Modulation of dendritic cell function by naive and regulatory CD4+T cells. J. Immunol. 176, 6202-10 (2006)

5. Mauri, C. \& Bosma, A. Immune regulatory function of B cells. Annu. Rev. Immunol. 30, 221-41 (2012)

6. Morva, A., et al. Maturation and function of human dendritic cells are regulated by B lymphocytes. Blood 119, 106-14 (2012).

7. Maddur, M. S., Kaveri, S. V. \& Bayry, J. Regulation of human dendritic cells by B cells depends on the signals they receive. Blood 119, 3863-4 (2012).

8. Maddur, M. S. et al. Human B cells induce dendritic cell maturation and favour Th2 polarization by inducing OX-40 ligand. Nat. Commun. 5, 4092 (2014).

9. Moens, L., Kane, A. \& Tangye, S. G. Naïve and memory B cells exhibit distinct biochemical responses following BCR engagement. Immunol. Cell Biol. 94, 774-86 (2016).

10. Tangye, S. G., Avery, D. T., Deenick, E. K. \& Hodgkin, P. D. Intrinsic differences in the proliferation of naive and memory human $B$ cells as a mechanism for enhanced secondary immune responses. J. Immunol. 170, 686-94 (2003).

11. Deenick, E. K. et al. Naive and memory human B cells have distinct requirements for STAT3 activation to differentiate into antibody-secreting plasma cells. J. Exp. Med. 210, 2739-53 (2013).

12. Sarvaria, A., Madrigal, J. A. \& Saudemont, A. B cell regulation in cancer and antitumor immunity. Cell. Mol. Immunol. 14, 662-74 (2017).

13. Darce, J. R., Arendt, B. K., Wu, X. \& Jelinek, D. F. Regulated expression of BAFFbinding receptors during human B cell differentiation. J. Immunol. 179, 7276-86 (2007). 